\title{
A smart-device based secondary prevention program for cerebrovascular disease patients: a randomized trial
}

\section{Francesco Motolese}

Università Campus Bio-Medico

Fioravante Capone ( $\square$ f.capone@policlinicocampus.it )

Università Campus Bio-Medico

Alessandro Magliozzi

Università Campus Bio-Medico

\section{Carlo Vico}

Università Campus Bio-Medico

\section{Gianmarco laccarino}

Università Campus Bio-Medico

\section{Emma Falato}

Università Campus Bio-Medico

\section{Fabio Pilato}

Università Campus Bio-Medico

Vincenzo Di Lazzaro

Università Campus Bio-Medico

\section{Research Article}

Keywords:

Posted Date: February 9th, 2022

DOI: https://doi.org/10.21203/rs.3.rs-1251620/v1

License: (1) (1) This work is licensed under a Creative Commons Attribution 4.0 International License. Read Full License 


\section{Abstract}

Background: Commercially available health devices are always more common and represent a unique chance to monitor patients for prolonged periods. This study aimed to test the feasibility of a smart device-based secondary prevention program in a cohort of patients with undetermined aetiology cerebrovascular disease.

Methods: Patients with non-disabling ischemic stroke and transient ischemic attack in the subacute phase were provided with a smartwatch and smart-devices to monitor various vital parameters for a 4week period (watch group). This group was compared with a standard-of-care group. This clinical trial was registered with ClinicalTrials.gov, ID: NCT04282993.

Results: In total, 161 patients were recruited, 87 in the Watch group and 74 in the control group. In the watch group, more than $90 \%$ of patients recorded the ECG at least once a day. In total, 5335 ECGs were recorded during the study. The median value of blood pressure measurements was 132 over $78 \mathrm{mmHg}$. The median oxygen saturation value was $97 \%$. From a clinical standpoint, nine AF episodes $(10,3 \%)$ in the Watch group - vs $3(4 \%)$ in the control group - were detected.

Conclusion: Our study suggests that prevention programs for cerebrovascular disease may benefit from the implementation of new technologies.

\section{Introduction}

Stroke is the major contributor to disability-adjusted life years (DALY) and the leading cause of death among neurological disorders ${ }^{1}$. The prevalence of cerebrovascular disease is expected to increase in the next decades because of population ageing ${ }^{2}$, while the stroke-related mortality has decreased considerably thanks to the improvement of acute phase management ${ }^{2}$. Antithrombotic therapies and adequate prevention strategies represent the mainstay of treatment for avoiding recurrences.

Despite the recent advances in secondary prevention, the rate of stroke recurrence at five years ranges from 10 to $20 \%$ of patients according to different studies, and it has not substantially changed in the last decade ${ }^{3,4}$. Besides, some stroke subtypes (e.g., cardioembolic) have an increased recurrence risk compared to others, suggesting that a tailored prevention program in this subgroup of patients may be advisable ${ }^{4}$. Recurrent strokes are associated with a longer hospital stay, higher mortality, and a higher degree of disability ${ }^{5}$.

Undefined strokes are at the highest risk of recurrences ${ }^{4}$. The actual incidence is debated, but many studies have reported that about a third of all strokes are without a clear aetiology after the standard diagnostic workup ${ }^{6-8}$. Among undefined strokes, embolic stroke of undetermined source (ESUS) indicates patients with non-lacunar strokes in which the most common cardioembolic sources have been 
excluded. ESUS accounts for about $17 \%$ of all strokes and is associated with a significant recurrence rate despite adequate antiplatelet treatment ${ }^{9}$.

Accordingly, specific management for cryptogenic strokes and ESUS still represent a debated field in clinical practice ${ }^{10}$. New tools as smartphones and smart devices are becoming increasingly available and can record different kinds of health data such as heart rate (HR) and motion but also electrocardiogram (ECG), blood pressure (BP) or peripheral oxygen saturation ${ }^{11}$. This represents a unique chance to monitor patients' health for prolonged periods outside the hospital setting, especially those affected by chronic diseases or for improving cardiovascular diseases prevention.

The present study aims to test the feasibility of a smart devices-based secondary prevention program in a cohort of patients with undefined aetiology cerebrovascular disease.

\section{Methods}

The study population included patients with non-disabling ischemic stroke - defined as a National Institutes of Health Stroke Scale (NIHSS) score $<3^{12}$ - or transient ischemic attack (TIA) in which the usual diagnostic workup - i.e. CT or MRI angiography of carotid arteries and intracranial vessels, prolonged ECG-monitoring, echocardiography - had not revealed any pathological alteration. This study was conducted accordingly to the declaration of Helsinki and was approved by the Ethics Committee of Università Campus Bio-Medico di Roma (Rome, Italy). Each participant gave a written informed consent before entering the study. This clinical trial was registered with ClinicalTrials.gov, NCT04282993 $(25 / 02 / 2020)$, and is completed and closed for participants.

Patients were recruited from our neurology department in the period from September 2020 to April 2021. We gathered the following baseline data: age, sex, education, complete medical history - including family history, previous cardiovascular events and risk factors assessment - and the NIHSS or ABCD ${ }^{2}$ score upon patient arrival at the emergency department. A history of atrial fibrillation (AF) or cognitive impairment as indicated by a Mini-Mental State Examination (MMSE) $<27$ were exclusion criteria. All patients were on antiplatelet therapy at the enrolment, presented a similar risk of stroke recurrence - as ascertained through the Essen Score ${ }^{13}$ - and received education about the secondary prevention of cerebrovascular disease. All patients were screened for dyslipidaemia. Participants were then randomly assigned to the smartwatch-based prevention program- i.e. WATCH group - or to the standard of care group - i.e. CONTROL group. As routine practice, all patients were scheduled with a follow-up appointment roughly one-month after the acute event. Besides, patients of the WATCH group received a smartwatch - i.e. Apple Watch Series 4 (Apple Inc., Cupertino, USA) - capable of reliably detecting AF through a single-lead ECG ${ }^{14}$. They also received a Bluetooth blood pressure monitor (iHealth BP7 wireless wrist BP monitor, iHealth Labs Inc., San Francisco, USA), a pulse oximeter (iHealth Air, iHealth Labs Inc., San Francisco, USA) and a smartphone - i.e. Apple iPhone series 6 (Apple Inc., Cupertino, USA) - to store the data collected during the 4-week observation period. Patients of the WATCH group were instructed to perform ECGs and to measure blood pressure and oxygen saturation multiple times during the day, especially 
whenever they did not feel well - e.g. in case of shortness of breath, palpitations, chest discomfort, etc. (Figure 1). This study followed the CONSORT guidelines for clinical trials.

\section{Smart devices}

The Smartwatch used in the study was equipped with various sensors. The main two of them were the enhanced PhotoPlethysmoGram (PPG) and the "heart sensor" to record ECG. The former contains a lightemitting diode (LED) that illuminates the skin and a diode measuring changes in light absorption caused by blood flow, thus recording the heartbeat rate (HR) and heartbeat variability - i.e., the interval between heartbeats in milliseconds (ms). The Apple Watch Series 4 was the first mass-produced consumer accessory receiving FDA approval for its Electrocardiogram (ECG) ${ }^{15}$. The heart sensor comprises two electrodes integrated on the backside of the watch and a third one in the digital crown. This allows customers to take a single-lead ECG from their wrist, just by holding their index finger on the digital crown for an adequate amount of time (i.e., 30 seconds). Then the recording is classified as atrial fibrillation, sinus rhythm, low or high HR, inconclusive, or poor quality by the embedded ECG app, which accordingly sends a notification to the user. A PDF of the recording is also stored on the smartphone app "Health" (Apple Inc., Cupertino, USA). The smartwatch also included other sensors like the gyroscope, the accelerometer - that records arm motion - and a GPS tracker, allowing accurate footsteps count.

The blood pressure monitor and the pulse oximeter worked through the app "iHealth My Vitals" (iHealth Labs, Inc., Mountain View, USA). The latter allows the customers to navigate among the available devices and measure the BP or Oxygen saturation, providing visual instruction on how to use the devices. These measures are automatically stored on the "Health" app of the smartphone.

At enrolment, patients in the WATCH group received the devices and the smartphone to store the data. This kit was controlled remotely through a mobile device management - ZuluDesk (Jamf, Minneapolis, USA) - to supervise devices, lock in the app store and limit internet access. However, personal data stored in the "Health" app on the smartphone were not accessible remotely, thus all the measurements performed by the patient were reviewed offline at the end of the study.

During enrolment, patients and caretakers received a 30-minute training on how to use devices and solve the most common technical issues. They also received a brief instructions booklet and an email address to contact in case of problems.

Patients returned the devices at the end of the study period - i.e., after four weeks. Anonymized data were then individually exported from the "Health" app as .eXtensible Markup Language (.xml) files and then converted to comma-separated values (.csv) files. Using a custom-made semiautomatic script for Matlab (The MathWorks Inc., Massachusetts, USA, Version: R2020a), data were pre-processed and parameters of interest - that include HR, heart rate variability (HRV), step count, BP values, Oxygen saturation - were analyzed. Regarding ECG, the app "Health" stored all the data and PDFs of the ECG taken by the patients. The EGCs labelled as "Atrial Fibrillation" were then visually reviewed by one of the authors (F.M., A.M., C.V) to confirm the diagnosis. 


\section{Statistical Analysis}

All statistical analyses were carried out using the Statistical Package for Social Science (SPSS) program, version 25.0 (IBMCo., Armonk, NY) and Matlab (The MathWorks Inc., Massachusetts, USA, Version:

R2020a). Data were expressed as mean or median [ $\pm S D$ and quartile ranges respectively] for continuous variables or as frequencies ( $n, \%)$ for categorical data. The Mann-Whitney rank-sum test and the $\chi^{2}$ test or Fisher's exact test were used to compare variables. Statistical significance was defined as $p$-value $<0.05$.

\section{Results}

A total of 210 patients were enrolled in this study. Forty-nine were excluded due to prespecified exclusion criteria - i.e., twenty-two patients presented an AF episode during hospital stay, thirteen did not meet the MMSE cut-off, fifteen refused to participate. One-hundred eleven had non-disabling strokes and fifty were TIA. Eighty-seven patients were assigned to the watch group and seventy-four to the standard group. All patients completed the 4-week program, and no dropout was reported. The main demographic and clinical characteristics are summoned up in Table 1. 
Table 1

Baseline characteristics of the Study Population

\begin{tabular}{|c|c|c|c|}
\hline & $\begin{array}{l}\text { WATCH GROUP } \\
(n=87)\end{array}$ & $\begin{array}{l}\text { STANDARD GROUP } \\
(n=74)\end{array}$ & $\begin{array}{l}\mathrm{p} \\
\text { value }\end{array}$ \\
\hline Age, median (Q1-Q3), y & $70(70-76)$ & $70(66-77,5)$ & $\begin{array}{l}\mathrm{p}= \\
0.352\end{array}$ \\
\hline Women, N (\%) & $77(47,8)$ & $84(52,2)$ & $\begin{array}{l}p= \\
0.903\end{array}$ \\
\hline Education & & & $\begin{array}{l}\mathrm{p}= \\
0.577\end{array}$ \\
\hline Higher education, n (\%) & $27(31)$ & $20(27)$ & \\
\hline Secondary or lower, n (\%) & $60(69)$ & $54(73)$ & \\
\hline Stroke, N (\%) & $63(72,4)$ & $48(64,9)$ & \\
\hline NIHSS, median (Q1-Q3) & $1(1-3)$ & $2(1-3)$ & $\begin{array}{l}\mathrm{p}= \\
0.087\end{array}$ \\
\hline TIA, N (\%) & $24(27,6)$ & $26(35,1)$ & \\
\hline ABCD 2 score, median (Q1-Q3) & $4(3-5)$ & $0(0-2)$ & $\begin{array}{l}\mathrm{p}< \\
0.001\end{array}$ \\
\hline Essen Score, median (Q1-Q3) & $2,5(1-4)$ & $3(2-4)$ & $\begin{array}{l}p= \\
0.130\end{array}$ \\
\hline Arterial Hypertension, N (\%) & $51(58,6)$ & $40(54,1)$ & $\begin{array}{l}p= \\
0.563\end{array}$ \\
\hline Diabetes, N (\%) & $12(16,2)$ & $19(21,8)$ & $\begin{array}{l}\mathrm{p}= \\
0.370\end{array}$ \\
\hline \multicolumn{4}{|l|}{ Dyslipidemia } \\
\hline LDL (mg/dl), mean (SD) & $119,4(39,2)$ & $112,4(36,6)$ & $\begin{array}{l}\mathrm{p}= \\
0.308\end{array}$ \\
\hline Triglycerides (mg/dl), mean (SD) & $130,8(73,7)$ & $114,9(50,4)$ & $\begin{array}{l}\mathrm{p}= \\
0.301\end{array}$ \\
\hline Smokers, N (\%) & $19(21,8)$ & $24(32,4)$ & $\begin{array}{l}\mathrm{p}= \\
0.246\end{array}$ \\
\hline Previous smokers, N (\%) & $20(23)$ & $18(24,3)$ & \\
\hline OSAS, N (\%) & $12(13,8)$ & $17(23)$ & \\
\hline
\end{tabular}

Q1: first quartile, Q3: third quartile, SD: standard deviation, NIHSS: National Institutes of Health Stroke Scale, TIA: Transient Ischemic Attack, LDL: Low-Density Lipoprotein, OSAS: Obstructive Sleep Apnea Syndrome, BMI: Body Mass Index 


\begin{tabular}{|c|c|c|c|}
\hline & $\begin{array}{l}\text { WATCH GROUP } \\
(\mathrm{n}=87)\end{array}$ & $\begin{array}{l}\text { STANDARD GROUP } \\
(n=74)\end{array}$ & $\begin{array}{l}p \\
\text { value }\end{array}$ \\
\hline BMI, mean (SD) & $26,2(4,4)$ & $26,2(5,6)$ & $\begin{array}{l}p= \\
0.617\end{array}$ \\
\hline Prior cerebrovascular disease, $\mathrm{N}(\%)$ & $17(19,5)$ & $13(17,6)$ & $\begin{array}{l}p= \\
0.847\end{array}$ \\
\hline $\begin{array}{l}\text { Family history of cardio- or cerebrovascular } \\
\text { disease, } \mathrm{N}(\%)\end{array}$ & $42(48,3)$ & $39(52,7)$ & $\begin{array}{l}p= \\
0.308\end{array}$ \\
\hline \multicolumn{4}{|c|}{$\begin{array}{l}\text { Q1: first quartile, Q3: third quartile, SD: standard deviation, NIHSS: National Institutes of Health Stroke } \\
\text { Scale, TIA: Transient Ischemic Attack, LDL: Low-Density Lipoprotein, OSAS: Obstructive Sleep Apnea } \\
\text { Syndrome, BMI: Body Mass Index }\end{array}$} \\
\hline
\end{tabular}

The median NIHSS was 1 (Q1-Q3: 1-3) for patients in the WATCH group and 2 (Q1-Q3: 1-3) for patients in the control group. Regarding $A B C D^{2}$, the median score for the WATCH group was 4 (Q1-Q3: 3-5) vs 0 (Q1Q3: 0-2). As said before, the two groups were similar in terms of Essen Score - median score WATCH GROUP 2.5 (Q1-Q3: 1-4) vs 3 (Q1-Q3: 2-4). Subjects of both groups shared the same cardiovascular risk profile (i.e., smoke, hypertension, diabetes, lipid-related blood markers, OSAS, BMI, history) $-p>0.05$. Age and gender distribution was also similar between groups.

In the WATCH group, sixty-three patients $(90,8 \%)$ recorded the ECG at least once a day. Among them, forty-one patients $(47,1 \%)$ of the entire cohort recorded the ECG twice a day (Figure 2). Eight patients $(9,2 \%)$ were not able to record ECGs. In total, 5335 ECGs - including inconclusive recordings $(n=1650$, $30,9 \%$ ), i.e., of low-quality or too short to be analyzed by the algorithm - were recorded during the study. On average, every patient recorded about 61 ECGs during the 4-week study period. Thirty-six $(41,4 \%)$ and twenty-six $(29,9)$ patients measured blood pressure and oxygen saturation twice a day, respectively (Figure 2). The median value of blood pressure measurements was 132 (Q1-Q3: 123-147) mmHg for systolic and 78 (Q1-Q3: 70-87) mmHg for diastolic. During the study, 9822 blood pressure measurements were attempted, of which 9558 (97.3\%) were valid. Oxygen saturation was measured 4089 times and 3499 (88.2\%) measures resulted valid. 85 (2.4\%) measures were removed from the analysis because below $85 \%$ in the absence of reported symptoms - we assumed these values might be due to technical issues. The median oxygen saturation value was 97 (Q1-Q3: 95-98) \%. The median number of steps per day was 3719 (Q1-Q3: 1663-9154) as recorded by the smartwatch or the smartphone. During the study period, the smartwatch recorded 207004 HR measures. The HR median value was 68 (Q1-Q3: 59-78) bpm, while the minimum and maximum values recorded were 28 and $195 \mathrm{bpm}$, respectively. The smartwatch also measured HRV by monitoring the time interval between two consecutive heartbeats. In particular, the median value of the HRV was 24.29 (Q1-Q3: 16.73-36.41) ms. These parameters recorded during the study are summarized in Table 2 and figure 3. 
Table 2

Values of the parameters recorded by the devices in the WATCH group

\begin{tabular}{|llllll|}
\hline & MEDIAN & Q1 & Q3 & MEAN & SD \\
\hline Diastolic BP (mmHg) & 78 & 70 & 87 & 78.43 & 12.36 \\
\hline Systolic BP (mmHg) & 132 & 123 & 147 & 135.56 & 18.59 \\
\hline Heart Rate (bpm) & 68 & 59 & 78 & 70.04 & 15.62 \\
\hline Heart Rate Variability (ms) & 24.29 & 16.73 & 36.41 & 33.12 & 16.73 \\
Oxygen Saturation (\%) & 97 & 95 & 98 & 96.18 & 2.28 \\
\hline Nr. of Steps per day & 3719 & 1663 & 9154 & 8073 & 11489 \\
\hline BP: Blood pressure; Q1: first quartile; Q3: third quartile & & \\
\hline
\end{tabular}

From a clinical standpoint, in the WATCH group, one patient $(1,1 \%)$ had a recurrent cerebrovascular event and nine patients $(10,3 \%)$ had at least an episode of AF detected by the smartwatch app. In the CONTROL group, three patients (4\%) were diagnosed with AF during the observation period and no cases of recurrent stroke were reported. These differences were not statistically significant - nor for cerebrovascular events $(p=0.355)$ nor for AF episodes $(p=0.13)$.

\section{Discussion}

The potential for improving the management of neurological disorders of smart health devices is welldemonstrated by a vast body of literature ${ }^{11}$. Since the number of smart device owners is growing worldwide, this will probably lead to a revolution in the doctor-patient relationship. Smart devices are embedded with sensors that record many different parameters - like continuous motion data, HR and HRV ${ }^{11}$ - and numerous studies have shown that they may be helpful for monitoring functional status in Multiple Sclerosis ${ }^{16}$, for improving compliance and outcomes of rehabilitation in chronic stroke survivors ${ }^{17}$, for providing personalized support to people affected by dementia ${ }^{18}$, for a better characterization of tremor in Parkinson's disease and essential tremor ${ }^{19,20}$, just to mention a few. However, to the best of our knowledge, this is the first study in which an integrated smart devices bundle has been used to monitor stroke patients in the subacute phase, when the recurrence risk is higher.

Evidence suggests that stroke incidence might be reduced by $50 \%$ through an adequate prevention program for the comprehensive management of cardiovascular risk factors, such as hypertension, diabetes, obesity, physical inactivity, and heart diseases ${ }^{1}$. Considering the dramatic impact of stroke from an epidemiological standpoint, there is a clear need of improving prevention, especially for patients with mild strokes or TIA. 
In this regard, AF is the most common disorder of heart rhythm and affects about 40 million people worldwide ${ }^{21}$. It is associated with a 5 -fold risk of stroke ${ }^{22}$ and is estimated to be the cause or a contributing factor in $15-20 \%$ of strokes ${ }^{23}$. Starting oral anticoagulation (OAC) in patients suffering from $\mathrm{AF}$ reduces the risk of thromboembolic stroke by about $64 \%$, at the expense of an increased risk of bleeding ${ }^{24}$. Two recent clinical trials have failed to demonstrate a clinically significant advantage of OAC in ESUS patients, even if data suggests an indication for longer ECG monitoring in specific patients' subgroups - i.e. older than 75 years when $A F$ is actually more common ${ }^{25}$. The role of covert $A F$ in cryptogenic stroke is debated ${ }^{9}$. Randomized trials on prolonged ECG monitoring through an implantable loop-recorded (ILR), like CRYSTAL-AF ${ }^{26}$ or meta-analyses ${ }^{27}$, have shown a prevalence of AF up to $30 \%$ in ESUS patients ${ }^{28}$ and the EMBRACE study have shown that a 30-day event recorder detected a firstepisode AF in $16 \%$ of stroke patients in the intervention group. Prolonged monitoring may be time- and resource-consuming and, even if current stroke guidelines include a recommendation for post-discharge heart-rhythm monitoring ${ }^{29}$, there is no indication about how long this should be extended. In the absence of definite guidelines or reliable best practices, there is a clear need for innovative solutions to help clinicians tailoring prevention programs on patients' characteristics. In this regard, ECG-recording smart devices offer the chance to augment our ability to detect arrhythmias in a real-life scenario ${ }^{14}$.

In our cohort, although not statistically significant, the number of AF episodes was higher in the intervention group (10.3 vs 4\%), despite the short duration of the observation period. Smart devices are for sure capable of detecting AF episodes, being almost as reliable as conventional tools ${ }^{30}$, and they may also be used by the most vulnerable groups of population - such as the elderly - thanks to their user-friendliness. However, smartwatches provide only a single-lead ECG, and no information about AF episode duration is available. The latter may be critical for the eventual decision of starting OAC since many authors have questioned the actual role of short duration AF episodes in stroke pathogenesis ${ }^{31}$. Another frequent criticism is that older people or people with a lower level of instruction might have difficulties using these technologies. In our study, the vast majority of patients took at least one ECG per day $(>90 \%)$ and more than two-thirds of the recorded ECGs were of sufficient quality to be analyzed by the in-built smartwatch algorithm. Considering the variegate nature of our cohort in terms of age and instruction, it is reasonable to say that the current intellectual resources are enough for implementing new technologies in clinical practice, as also shown by other similar experiences ${ }^{32,33}$. Indeed, the recent Coronavirus Disease 2019 pandemic had the unexpected twist of prompting the implementation of remote monitoring and telemedicine programs around the world and the number of papers on this topic has skyrocketed in the last two years ${ }^{34}$.

At present, smartwatch customers can take an ECG at discrete times and with active engagement but, through the application of machine learning algorithms, it is also conceivable the passive detection of AF using smartwatch data ${ }^{35}$. This will make the implementation of health gadgets even easier in daily life. Other measures can be also valuable, such as HRV, corresponding to the physiological variation of the time interval between consecutive heartbeats. $\mathrm{HRV}$ is an index of autonomic nervous system functioning 
and is considered normal between 20 and $200 \mathrm{~ms}$. Some authors have suggested that increased HRV may predict the onset of $A F$, indicating an imbalance in the cardiac vagal tone ${ }^{36}$. HRV is one of the most promising biomarkers of AF and is recorded passively, thus its use in daily life scenarios deserves more research.

The other parameters investigated in this study included HR, BP, SaO2 and step count. The role of these measures is more evident at the individual rather than group level. For instance, patients who showed HR $<35 \mathrm{bpm}$ on multiple occasions were scheduled with a cardiologic evaluation. Or patients with lower levels of physical activity - the median value of daily footsteps was disappointingly low, i.e., 3700 steps/day - were advised to increase the time dedicated to exercise, highlighting the long-term benefit on cardiovascular fitness ${ }^{37}$. We have to acknowledge that patients have reported some technical issues using the $\mathrm{BP}$ and $\mathrm{SaO} 2$ monitors. These were third-part devices managed by a specifically designed app and sometimes users have experienced compatibility issues, e.g., Bluetooth pairing not working in several occasion. This was also the reason for the lower compliance we noted for BP and $\mathrm{SaO} 2$ measures. Integrated environments make the user experience smoother and might stimulate patients' engagement, thus improving compliance. In our opinion, the next step is to develop an integrated app capable of managing different devices and offering a better and simpler user experience. This specifically designed app should also provide an online connection with clinicians that might be informed in real-time of red flags, i.e., very low-HR or elevated BP. Alongside this real-time warning system, the other parameters HRV or step count - may be reviewed at different timescales to have the overall vision of how the patient is doing. One could also imagine specific training programs to increase compliance for sedentary patients that do not accomplish the required exercise targets. Or, in general, specific tips on how to improve lifestyle.

Remote monitoring programs - like the one we set up for this experiment - may be the occasion to actively engage patients in the therapeutic relationship. Indeed, despite our concerns - Italy is one of the European countries with the highest digital illiteracy ${ }^{38}$ - patients adherence to the study protocol was overall satisfying since $90 \%$ of patients recorded the ECGs at least once a day and the quality of data of the other parameters - such as $\mathrm{BP}, \mathrm{SaO} 2$ and steps - was satisfactory. There is still a long road ahead before the definitive implementation of these technologies in clinical practice. However, investing in technological ecosystems to improve patients' user experience and quality of recorded data would pay substantial returns in terms of better management of diseases and the reduction of the socioeconomic burden of healthcare costs on society. Another issue to deal with is the vast amount of data that can be recorded. At present, only a small fraction of it might have a substantial role in helping clinicians make informed clinical decisions. Handling big data is a great challenge for healthcare providers. Biometric sensors from wearables record a large volume of unstructured data that must be processed into meaningful structured information, keeping in mind the "Mantra" of big data analytics, i.e. the more the data, the more insight one can gain from them. This is necessary for improving our skills in making clinical predictions, providing early warnings of disease progression, finding novel biomarkers, just to mention a few ${ }^{39}$. Thus, it is mandatory to carefully choose the quality and quantity of data to provide to 
clinicians, without neglecting yet another issue, i.e., the importance of protecting our patients' data. Indeed, the safety of personal data is of paramount importance for the definitive implementation of new technologies in daily routine, but it is an often-overlooked concern.

This study has some limitations to be addressed. Firstly, the limited sample size was defined to verify the feasibility of our approach and not to investigate differences between the two groups. Secondly, the short duration of the study that was chosen to investigate the stroke subacute phase, in which the risk of recurrence is higher. However, as data from the literature show, it is not clear what is the optimal time window for ECG monitoring and, in our opinion, more extended observation periods may be useful thanks to smartwatch cost-effectiveness. Another limitation is the lack of data about sleep. After reviewing some of the most common apps for the iOS environment, we did not find anything that could add value to our study - considering we already recorded HR and HRV during sleep. Finally, we have to acknowledge the slight heterogeneity of the groups, which was probably due to the "real-life" study design, i.e. patients were recruited consecutively from our Clinic according to inclusion and exclusion criteria and then randomized. In our opinion, this latter point is also a strength of our study since it demonstrated that using new technologies is possible even with the current resources and the current digital literacy level of patients.

\section{Conclusion}

Our study suggests that prevention programs for cerebrovascular disease may benefit from the implementation of new technologies. Collecting real-life data may be helpful to identify red flags in patients at high risk of stroke, eventually prompting medical attention. The use of remote monitoring devices - allowing the continuous evaluation of different vital parameters - might revolutionize healthcare systems organizations ${ }^{11}$. However, more studies on a larger sample size are warranted to investigate the efficacy and cost-effectiveness of this approach against the standard of care.

\section{Declarations}

\section{Ethics and consent}

This study was conducted accordingly to the declaration of Helsinki and was approved by the Ethics Committee of Università Campus Bio-Medico di Roma (Rome, Italy). Each participant gave a written informed consent before entering the study. This clinical trial was registered with ClinicalTrials.gov, NCT04282993 (25/02/2020), and is completed and closed for participants.

\section{Acknowledgement}

This work was supported and funded by the "Associazione Nazionale fra le imprese Assicuratrici" (ANIA).

\section{Authors contribution}


FM, FC and VDL conceived and designed the study. AM, CV, GI recruited the patients and collected the data. FM, FC, EF, FP analyzed the data and contributed to data interpretation. FM wrote the first draft of the manuscript. FC, FP, VDL reviewed the manuscript. All authors approved the final manuscript for submission.

\section{Data availability}

The data that support the findings of this study are available on request from the corresponding author. The data are not publicly available because their containing information could compromise the privacy of research participants.

\section{Code availability}

The code used for the present study is available as supplementary material.

\section{Declaration of Competing Interest}

The author declared the following potential conflicts of interest with respect to the research, authorship, and/or publication of this article: F.C. has received travel grants from Biogen, Merck, Teva and SanofiGenzyme; FM, AM, CV, GI, EF, FP, VDL have no conflict of interest.

\section{References}

1. Brainin M, Feigin VL, Norrving B, et al (2020) Global prevention of stroke and dementia: the WSO Declaration. Lancet Neurol 19:487-488. https://doi.org/10.1016/S1474-4422(20)30141-1

2. Feigin VL, Vos T, Nichols E, et al (2020) The global burden of neurological disorders: translating evidence into policy. Lancet Neurol 19:255-265. https://doi.org/10.1016/S1474-4422(19)30411-9

3. Flach C, Muruet W, Wolfe CDA, et al (2020) Risk and Secondary Prevention of Stroke Recurrence: A Population-Base Cohort Study. Stroke 51:2435-2444.

https://doi.org/10.1161/STROKEAHA.120.028992

4. Rücker V, Heuschmann PU, O'Flaherty M, et al (2020) Twenty-Year Time Trends in Long-Term CaseFatality and Recurrence Rates after Ischemic Stroke Stratified by Etiology. Stroke 2778-2785. https://doi.org/10.1161/STROKEAHA.120.029972

5. Jerrgensen HS, Nakayama H, Reith J, et al (1997) Stroke recurrence: Predictors, severity, and prognosis. The Copenhagen Stroke Study. Neurology 48:891-895. https://doi.org/10.1212/WNL.48.4.891

6. Petty GW, Brown RD, Whisnant JP, et al (1999) Ischemic Stroke Subtypes. Stroke 30:2513-2516. https://doi.org/10.1161/01.STR.30.12.2513

7. Kolominsky-Rabas PL, Weber M, Gefeller O, et al (2001) Epidemiology of Ischemic Stroke Subtypes According to TOAST Criteria. Stroke 32:2735-2740. https://doi.org/10.1161/hs1201.100209 
8. Schulz UGR, Rothwell PM (2003) Differences in Vascular Risk Factors Between Etiological Subtypes of Ischemic Stroke. Stroke 34:2050-2059. https://doi.org/10.1161/01.STR.0000079818.08343.8C

9. Ntaios G (2020) Embolic Stroke of Undetermined Source. J Am Coll Cardiol 75:333-340. https://doi.org/10.1016/j.jacc.2019.11.024

10. Kasner SE, Swaminathan B, Lavados P, et al (2018) Rivaroxaban or aspirin for patent foramen ovale and embolic stroke of undetermined source: a prespecified subgroup analysis from the NAVIGATE ESUS trial. Lancet Neurol 17:1053-1060. https://doi.org/10.1016/S1474-4422(18)30319-3

11. Sim I (2019) Mobile Devices and Health. N Engl J Med 381:956-968. https://doi.org/10.1056/NEJMra1806949

12. Fischer U, Baumgartner A, Arnold M, et al (2010) What Is a Minor Stroke? Stroke 41:661-666. https://doi.org/10.1161/STROKEAHA.109.572883

13. Weimar C, Diener H-C, Alberts MJ, et al (2009) The Essen Stroke Risk Score Predicts Recurrent Cardiovascular Events. Stroke 40:350-354. https://doi.org/10.1161/STROKEAHA.108.521419

14. Foster KR, Torous J (2019) The Opportunity and Obstacles for Smartwatches and Wearable Sensors. IEEE Pulse 10:22-25. https://doi.org/10.1109/MPULS.2018.2885832

15. https://www.accessdata.fda.gov/cdrh_docs/pdf18/DEN180044.pdf

16. Midaglia L, Mulero P, Montalban X, et al (2019) Adherence and Satisfaction of Smartphone- and Smartwatch-Based Remote Active Testing and Passive Monitoring in People With Multiple Sclerosis: Nonrandomized Interventional Feasibility Study. J Med Internet Res 21:. https://doi.org/10.2196/14863

17. Chae SH, Kim Y, Lee K-S, Park H-S (2020) Development and Clinical Evaluation of a Web-Based Upper Limb Home Rehabilitation System Using a Smartwatch and Machine Learning Model for Chronic Stroke Survivors: Prospective Comparative Study. JMIR mHealth uHealth 8:e17216. https://doi.org/10.2196/17216

18. Thorpe J, Forchhammer BH, Maier AM (2019) Adapting Mobile and Wearable Technology to Provide Support and Monitoring in Rehabilitation for Dementia: Feasibility Case Series. JMIR Form Res 3:e12346. https://doi.org/10.2196/12346

19. López-Blanco R, Velasco MA, Méndez-Guerrero A, et al (2019) Smartwatch for the analysis of rest tremor in patients with Parkinson's disease. J Neurol Sci 401:37-42. https://doi.org/10.1016/j.jns.2019.04.011

20. Barrantes S, Sánchez Egea AJ, González Rojas HA, et al (2017) Differential diagnosis between Parkinson's disease and essential tremor using the smartphone's accelerometer. PLoS One 12:e0183843. https://doi.org/10.1371/journal.pone.0183843

21. Kornej J, Börschel CS, Benjamin EJ, Schnabel RB (2020) Epidemiology of Atrial Fibrillation in the 21st Century. Circ Res 127:4-20. https://doi.org/10.1161/CIRCRESAHA.120.316340

22. Wolf PA, Abbott RD, Kannel WB (1991) Atrial fibrillation as an independent risk factor for stroke: the Framingham Study. Stroke 22:983-988. https://doi.org/10.1161/01.STR.22.8.983 
23. Reiffel JA (2014) Atrial Fibrillation and Stroke: Epidemiology. Am J Med 127:e15-e16. https://doi.org/10.1016/j.amjmed.2013.06.002

24. Kotalczyk A, Mazurek M, Kalarus Z, et al (2021) Stroke prevention strategies in high-risk patients with atrial fibrillation. Nat Rev Cardiol 18:276-290. https://doi.org/10.1038/s41569-020-00459-3

25. Diener H-C, Sacco RL, Easton JD, et al (2019) Dabigatran for Prevention of Stroke after Embolic Stroke of Undetermined Source. N Engl J Med 380:1906-1917. https://doi.org/10.1056/NEJMoa1813959

26. Sanna T, Diener H-C, Passman RS, et al (2014) Cryptogenic Stroke and Underlying Atrial Fibrillation. N Engl J Med 370:2478-2486. https://doi.org/10.1056/NEJMoa1313600

27. Sposato LA, Cipriano LE, Saposnik G, et al (2015) Diagnosis of atrial fibrillation after stroke and transient ischaemic attack: a systematic review and meta-analysis. Lancet Neurol 14:377-387. https://doi.org/10.1016/S1474-4422(15)70027-X

28. Kamel H, Merkler AE, ladecola C, et al (2019) Tailoring the Approach to Embolic Stroke of Undetermined Source. JAMA Neurol 76:855. https://doi.org/10.1001/jamaneurol.2019.0591

29. Kernan WN, Ovbiagele B, Black HR, et al (2014) Guidelines for the Prevention of Stroke in Patients With Stroke and Transient Ischemic Attack. Stroke 45:2160-2236. https://doi.org/10.1161/STR.0000000000000024

30. Perez M V., Mahaffey KW, Hedlin H, et al (2019) Large-Scale Assessment of a Smartwatch to Identify Atrial Fibrillation. N Engl J Med 381:1909-1917. https://doi.org/10.1056/NEJMOA1901183/SUPPL_FILE/NEJMOA1901183_DATA-SHARING.PDF

31. Tirschwell D, Akoum N (2021) Detection of Subclinical Atrial Fibrillation After Stroke. JAMA 325:2157. https://doi.org/10.1001/jama.2021.7429

32. Marano M, Motolese F, Rossi M, et al (2021) Remote smartphone gait monitoring and fall prediction in Parkinson's disease during the COVID-19 lockdown. Neurol Sci 42:. https://doi.org/10.1007/s10072-021-05351-7

33. Motolese F, Magliozzi A, Puttini F, et al (2020) Parkinson's Disease Remote Patient Monitoring During the COVID-19 Lockdown. Front Neurol 11:1190. https://doi.org/10.3389/fneur.2020.567413

34. Telehealth: A post-COVID-19 reality? | McKinsey. https://www.mckinsey.com/industries/healthcaresystems-and-services/our-insights/telehealth-a-quarter-trillion-dollar-post-covid-19-reality. Accessed 18 Nov 2021

35. Tison GH, Sanchez JM, Ballinger B, et al (2018) Passive Detection of Atrial Fibrillation Using a Commercially Available Smartwatch. JAMA Cardiol 3:409.

https://doi.org/10.1001/jamacardio.2018.0136

36. Vikman S, Mäkikallio TH, Yli-Mäyry S, et al (2003) Heart rate variability and recurrence of atrial fibrillation after electrical cardioversion. Ann Med 35:36-42. https://doi.org/10.1080/07853890310004110

37. Tian D, Meng J (2019) Exercise for Prevention and Relief of Cardiovascular Disease: Prognoses, Mechanisms, and Approaches. Oxid Med Cell Longev 2019:1-11. 
https://doi.org/10.1155/2019/3756750

38. https://www.bloomberg.com/news/articles/2020-07-17/limited-digital-literacy-is-bad-news-for-italys-youth-map

39. Dash S, Shakyawar SK, Sharma M, Kaushik S (2019) Big data in healthcare: management, analysis and future prospects. J Big Data 201961 6:1-25. https://doi.org/10.1186/S40537-019-0217-0

\section{Figures}




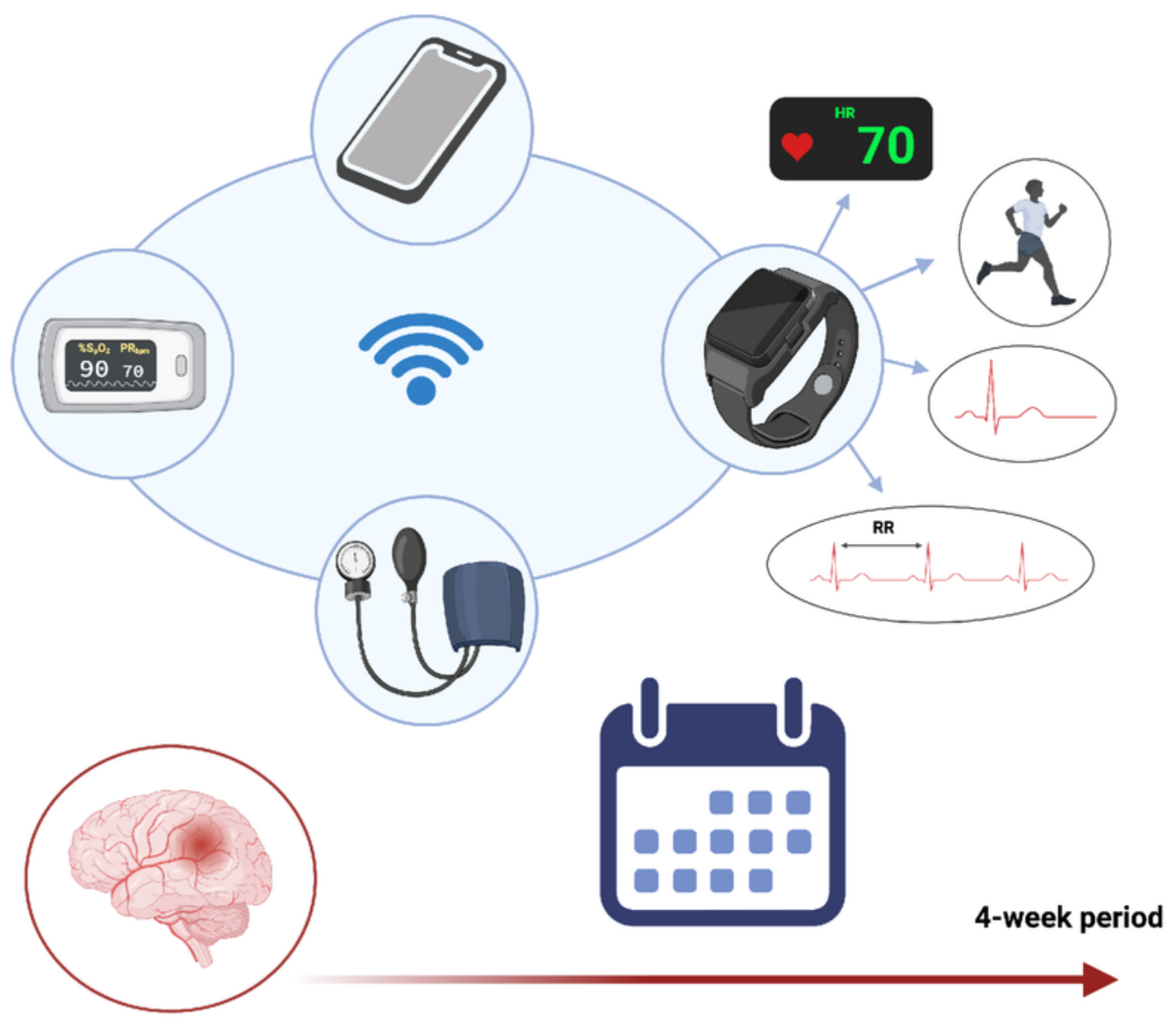

\section{Figure 1}

\section{WATCH-group study kit}

Patients enrolled in the WATCH group received a smartwatch - capable of recording heart rate, heart rate variability, footsteps, and ECG (the latter requires active participation of patients) - and a wireless blood pressure monitor and pulsi-oximeter. Data were individually collected on a smartphone that was set up on 
the day of the enrolment. Patients also received instructions on how to use the devices, both orally and in written form. The study ended after four weeks.

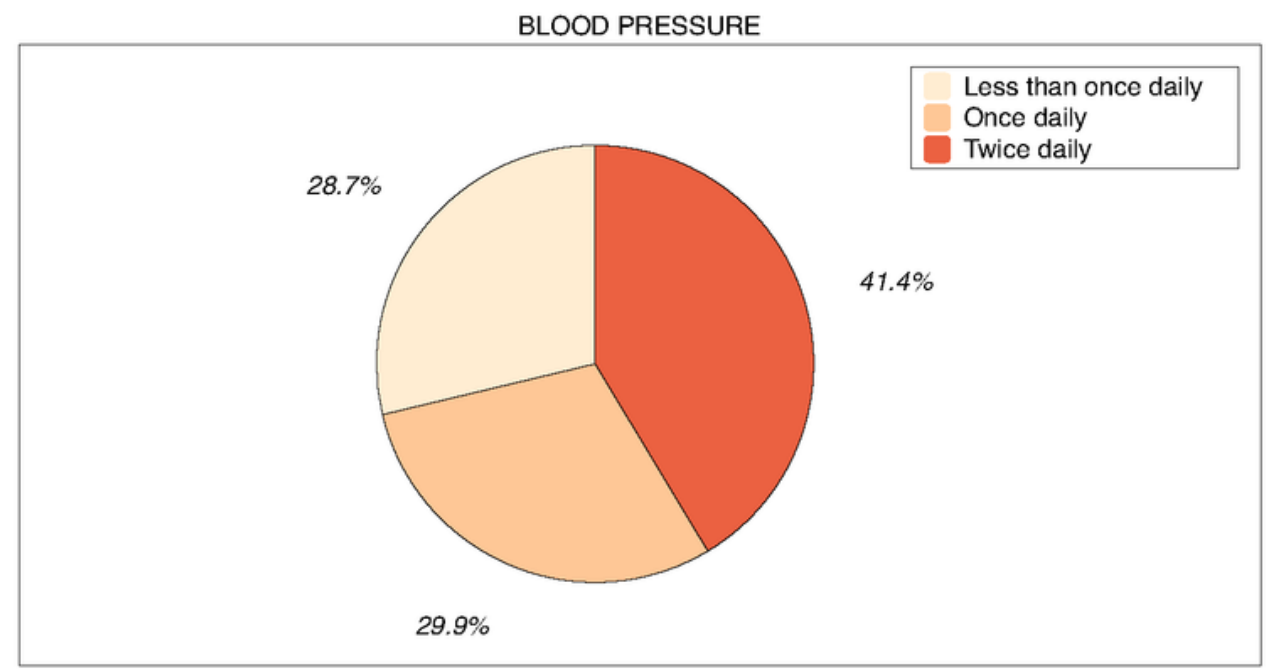

OXYGEN SATURATION

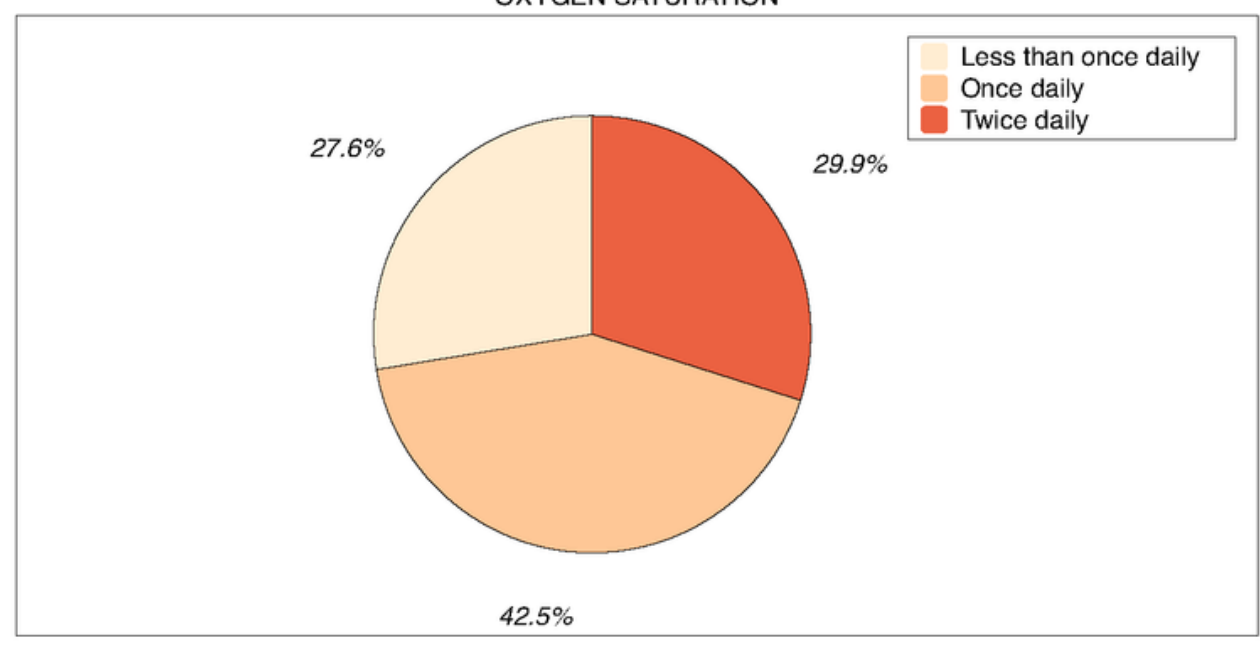

ECG

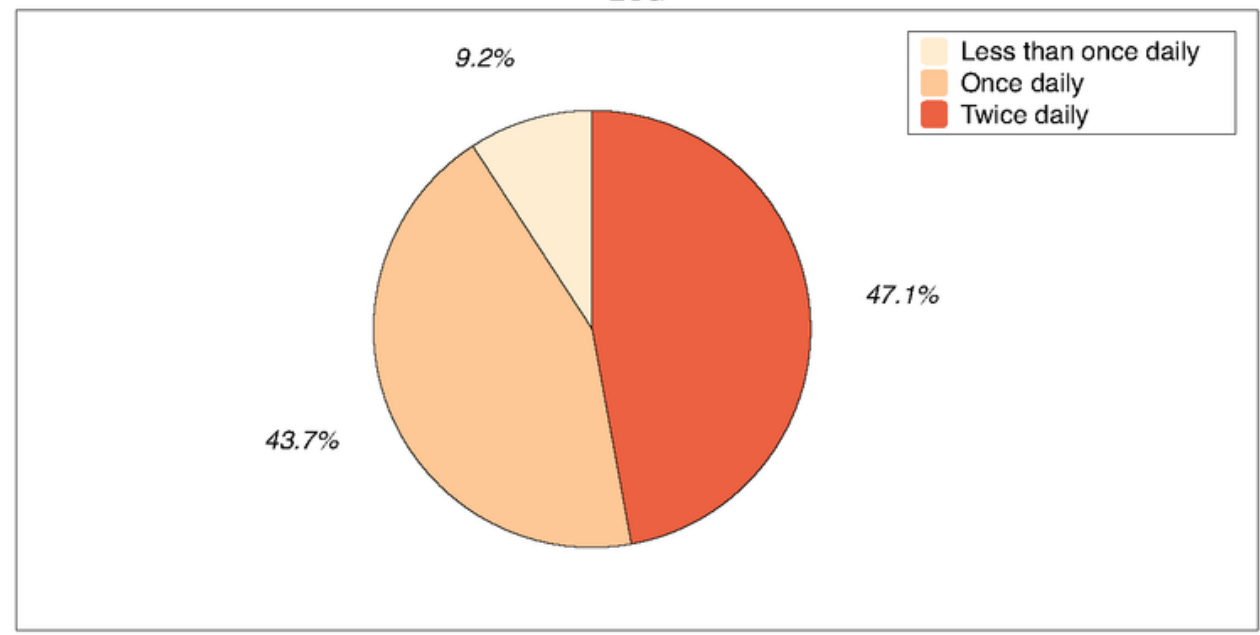

Figure 2 


\section{Pie charts depicting compliance during the study}

Patients of the WATCH group were asked to record ECG and measure blood pressure and oxygen saturation multiple times daily, especially whenever they did not feel well. If not able to perform any measurement or ECG recording, they were considered as "non-compliant".
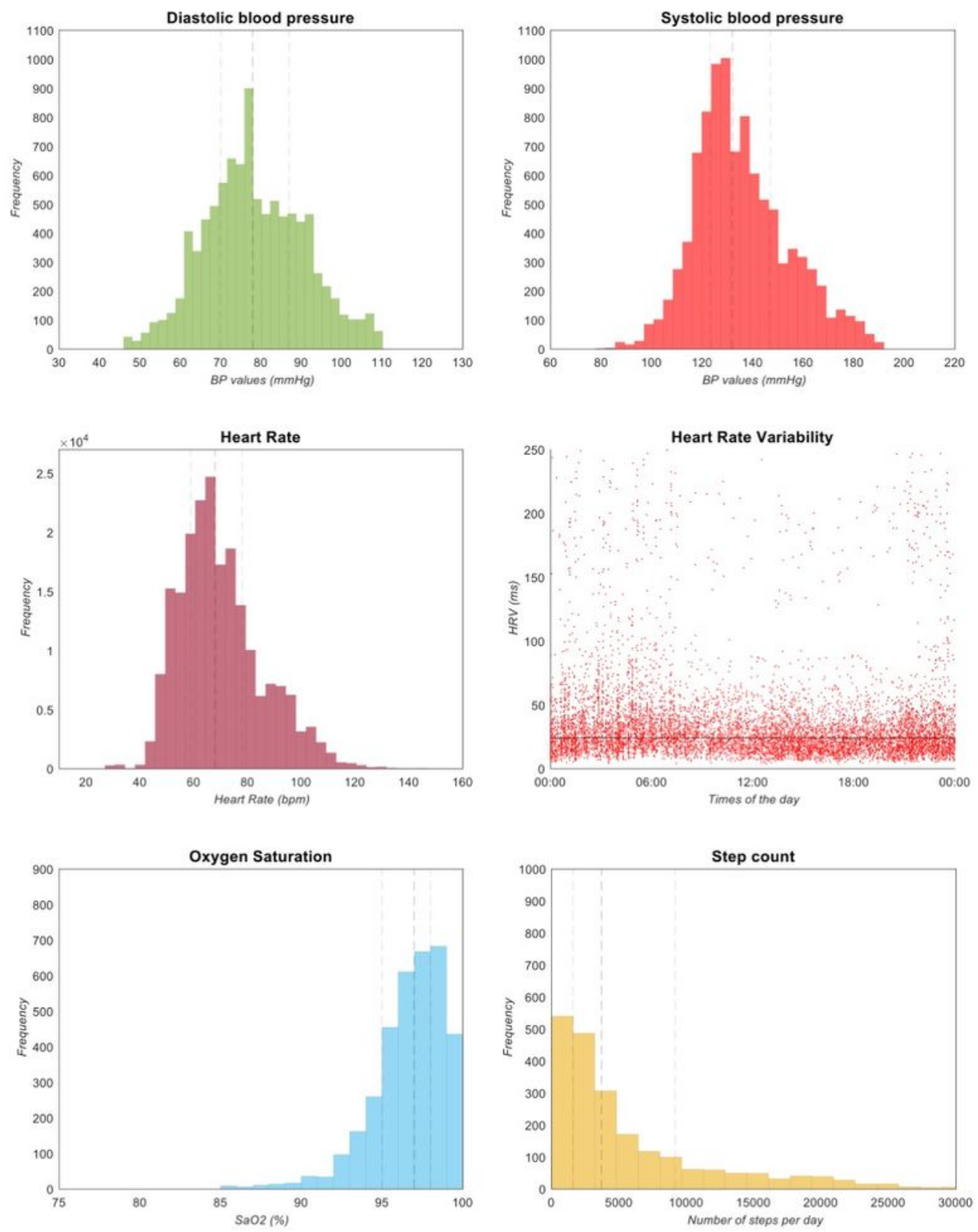
Figure 3

Parameters recorded by the devices in the WATCH-group

Frequency distribution of BP values, Saturation values and step count. A scatter plot of HRV in function of time of the day is also plotted. The dashed lines represent the median (black), the Q1 and Q3 (grey) values. Q1: first quartile; Q3: third quartile; BP: Blood Pressure; HRV: Heart Rate Variability; SaO2: Oxygen Saturation. 